\title{
Conjugately-Matched Uniaxial Metamaterials Make Extremely Efficient Absorbers, Emitters, and Reflectors
}

\author{
C. A. Valagiannopoulos ${ }^{1}$, C. R. Simovski ${ }^{2}$, and S. A. Tretyakov ${ }^{2}$ \\ ${ }^{1}$ Department of Physics, School of Science and Technology, Nazarbayev University, \\ Qabanbay Batyr Ave 53, Astana, KZ-010000, Kazakhstan. \\ ${ }^{2}$ Department of Radio Science and Engineering, School of Electrical Engineering, Aalto University, \\ P.O. 13000, FI-00076, Aalto, Finland. \\ konstantinos.valagiannopoulos@nu.edu.kz
}

\begin{abstract}
The speed with which electromagnetic energy can be wirelessly transferred from a source to the user is a crucial parameter for performance of a large number of electronic and photonic devices. In this presentation we determine the constituent parameters of a medium which supports theoretically infinite energy concentration close to a medium sample surface; such a material combines properties of Perfectly Matched Layers (PML) and Double-Negative (DNG) media. It realizes conjugate matching with free space for every possible mode including, most importantly, all evanescent modes. We show that extremely high-amplitude resonating fields in the vicinity of a conjugately matched body can create large far-field radiation with the use of randomly placed particles which play the role of emission "vessels".
\end{abstract}

\section{GENERAL PRINCIPLES}

In order to maximize the power wirelessly delivered from a source to a load, the load should be conjugately matched to the internal impedance of the source. This well-known maximal power principle applied in circuits can be generalized to cover electrically sizable electromagnetic structures. The only difference is that in the latter case there are infinitely many channels (modes) for transferring energy; if all of them obey the conjugate-matching principle, the tranferred power $P$ is diverging [1]. In particular, the load can be replaced by a semi-infinite halfspace filled with a uniaxial medium of relative constituent properties $\left(\varepsilon_{r t}, \mu_{r t}, \varepsilon_{r n}\right)$ and the source by any dipole or multipole placed in the vicinity of the interface [2]. Considering TM illumination (which does not damage the generality), the internal impedance of the source is the one of free space: $Z_{0}=-j \frac{\eta_{0}}{k_{0}} \sqrt{k_{t}^{2}-k_{0}^{2}}$, where $k_{0}=$ $2 \pi / \lambda_{0}=2 \pi f \sqrt{\varepsilon_{0} \mu_{0}}$ is the free-space wavenumber and $k_{t}$ the transverse wavenumber for the direction parallel to the interface ( $t$ stands for transverse and $n$ for normal direction to the surface of the material sample). The symbols $\eta_{0}, \lambda_{0}, \varepsilon_{0}$ and $\mu_{0}$ correspond to the free-space impedance, wavelength, permittivity and permeability, respectively ( $e^{+j 2 \pi f \tau}$ time dependence is suppressed, and $f$ is the operational frequency). The TM wave impedance of the uniaxial medium is given by: $Z=-j \frac{\eta_{0}}{k_{0} \varepsilon_{r t}} \sqrt{\frac{\varepsilon_{r t}}{\varepsilon_{r n}} k_{t}^{2}-\varepsilon_{r t} \mu_{r t} k_{0}^{2}}$, where $\left(\varepsilon_{r t}, \mu_{r t}\right)$ are the transverse and normal permittivities, and $\mu_{r t}$ its transverse permeability. It has been shown [2] that the constituent parameters of the uniaxial medium which can achieve conjugate matching with free space $Z\left(k_{t}\right)=Z_{0}^{*}\left(k_{t}\right)$ for every single mode $k_{t}$, should satisfy the Perfectly Matched Layer (PML) rule [3] but with negative real parts, namely:

$$
\varepsilon_{r t}=\mu_{r t}=\frac{1}{\varepsilon_{r n}}=a-j b, a<0 .
$$

That is why we call such an effective material Conjugate Matched Layer (CML). The ordinary PML just behaves like a perfect "black body" [4] based solely on the propagating modes; on the contrary, CML fully exploits all the evanescent waves additionally. Note that the parameter $b>0$ represents the losses along the transverse direction, which means that a medium defined by (1) is active along the normal direction $\left(\operatorname{Im}\left[\varepsilon_{r n}\right]>0\right)$. 


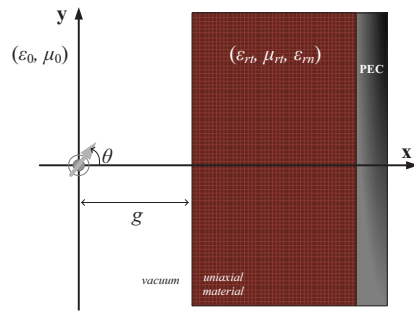

(a)

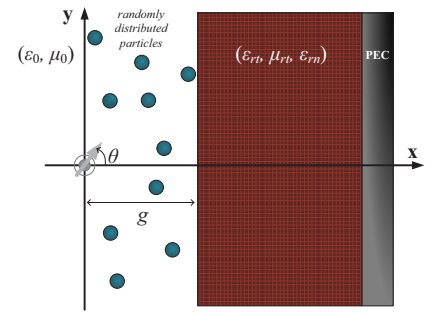

(b)

Fig. 1: The configurations offering: (a) extremely high absorbing power and (b) very high emitting power.

\section{NEAR-FiELD ENERGy TRANSFER}

If we particularize our research to the test-bed configuration of Fig. 1(a), namely, a grounded slab excited by a tilted (by angle $\theta$ ) electric-dipole line source (expression for the incident field of such source see e.g. in [5]) at distance $g$ from the interface, we can find an analytical expression for the electromagnetic power $P$ absorbed in the slab. To better understand better the variation of $P$, we consider a slightly perturbed version of the material (1) with $\varepsilon_{r n}=1 / \varepsilon_{r t}-j \delta=1 / \mu_{r t}-j \delta$ (for $\delta=0$, we have the perfect CML of (1)). The absorbed power $P$ can be written as a sum of two terms: one expressing the energy transferred via the propagating modes $P_{\text {prop }}$ (which is not dependent on $a, b, \delta$ ) and another corresponding to the evanescent modes $P_{\text {evan }}$. If we assume a small perturbation parameter $\delta \rightarrow 0, P_{\text {evan }}$ takes the following form [2]:

$$
P_{\text {evan }} \cong P_{\text {prop }} \frac{8|a|}{k_{0}^{2} \pi} \int_{k_{0}}^{+\infty} \frac{k_{t}^{2}\left(k_{t}^{2}-k_{0}^{2} \sin ^{2} \theta\right)}{\left(k_{t}^{2}-k_{0}^{2}\right)^{3 / 2}} e^{-2 g \sqrt{k_{t}^{2}-k_{0}^{2}}} \frac{\delta}{[1+\operatorname{sgn}(a)]^{2}+\delta^{2}\left[\frac{k_{t}^{2}\left|\varepsilon_{r t}\right|}{2\left(k_{t}^{2}-k_{0}^{2}\right)}\right]^{2}} d k_{t} .
$$

It is remarkable that for the DNG case $(a<0)$ the aforementioned quantity behaves like $P_{\text {evan }} \sim P_{\text {prop }} / \delta$, which means that it takes very high values when the uniaxial slab possesses properties close to those of a CML (1). Furthermore, it should be noted that the sign of the absorbed power $P_{\text {evan }}$ (and accordingly the overall power $P=P_{\text {prop }}+P_{\text {evan }}$ is negative when $\delta<0$; that means that the slab acts as a secondary source and pumps (extremely quickly) energy to the system. In other words, our load (a grounded uniaxial slab) can either absorb or emit energy infinitely fast, if it obeys the rule (1) and simultaneously is slightly lossy $(\delta>0)$ or slightly active $(\delta<0)$, respectively.

The extremely efficient absorption is demonstrated by Figs. 2, where the ratio $P / P_{\text {prop }}$ is represented as a function of $a$ for various loss parameters $b>0$. We consider two scenarios: one in Fig. 2(a), where the rule (1) is followed (not exactly but with a small $\delta>0$ ) and one in Fig. 2(b) where the effective parameters of the medium are all passive, namely $\varepsilon_{r t}=\mu_{r t}=a-j b$ but $\varepsilon_{r n}=\operatorname{Re}\left[1 / \varepsilon_{r t}\right]=a /\left(a^{2}+b^{2}\right)$. The most dominant characteristic of the two graphs is the switch of $P$ from very low values for $a>0$ (conventional PML "black body" absorption $\left.P_{\text {prop }}\right)$ to extremely high values in the CML regime $(a<0)$. It should be stressed that the nonactive case exhibits high absorbing efficiency $\left(P \gg P_{\text {prop }}\right)$ but smaller than the CML structure. Furthermore, an interesting feature is the effect of $\delta$ in Fig. 2(a) which makes $P$ to increase substantially for small $|a<0|$; on the contrary, in Fig. 2(b), where all material parameters are passive, the power $P$ is an increasing function of $|a<0|$.

\section{FAR-FIELD ENERGY TRANSFER}

When the structure exhibits extremely high efficiency as an emitter, namely has $P_{\text {evan }}, P \rightarrow-\infty$, which becomes feasible by choosing a small $|\delta<0|$, this does not mean that this huge power travels far away. Due to the nature of evanescent fields due to which $P_{\text {evan }}$ is developed, the produced signal decays exponentially with the distance from the planar interface. In order to remedy such a weakness and manage to send a significant portion of that huge $P$ (regardless of the choice of the sign of $\delta$ ) to the far region, we propose the configuration depicted in Fig. 1(b). In particular, we position a large number of randomly distributed particles in the near field of the structure. These particles are able to couple the evanescent fields with the propagating free-space modes and radiate far away from the interface. The considered boundary value problem can be treated with an integral equation 


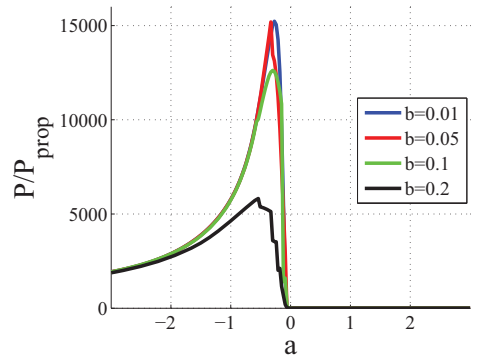

(a)

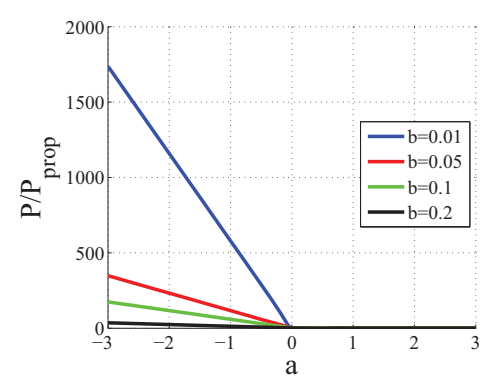

(b)

Fig. 2: The normalized absorbed power $P / P_{\text {prop }}$ as a function of $a=\operatorname{Re}\left[\varepsilon_{r t}\right]=\operatorname{Re}\left[\mu_{r t}\right]$ for several values of the loss parameter $b$ in: (a) CML passive case and (b) nonactive case.

formulation [6] which fully describes the wave interactions between the source, the CML slab and the cylindrical particles. In this way, one can evaluate the radiated power in the presence $\left(P_{\mathrm{rad}}\right)$ and the absence $\left(P_{\mathrm{rad}}^{\prime}\right)$ of the particles and accordingly compute the ratio $P_{\mathrm{rad}} / P_{\mathrm{rad}}^{\prime}$ which shows how significant is the radiation enhancement.

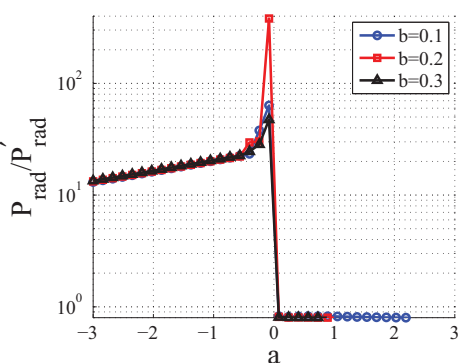

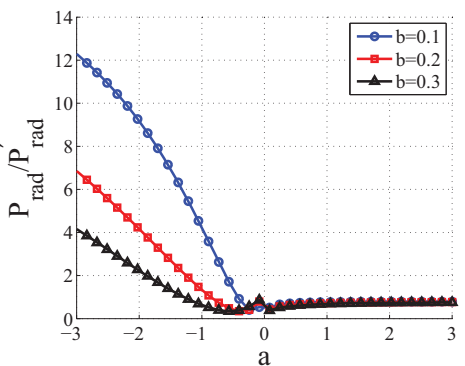

(b)

Fig. 3: The radiation enhancement ratio $P_{\mathrm{rad}} / P_{\mathrm{rad}}^{\prime}$ as a function of $a=\operatorname{Re}\left[\varepsilon_{r t}\right]=\operatorname{Re}\left[\mu_{r t}\right]$ for several losses $b$ in: (a) CML passive case and (b) nonactive case.

The dramatic effect of the particles located in the near field of the CML body is shown in Figs. 3, where the parameter $P_{\mathrm{rad}} / P_{\mathrm{rad}}^{\prime}$ is represented as a function of $a$ for various loss levels $b>0$. We consider two cases similar to those of Figs. 2 in the presence of numerous electrically small cylinders in the vicinity of the interface. Again the proposed solution works only for DNG media $(a<0)$ where extremely high values of the radiation enhancement ratio are observed for the CML case (Fig. 3(a)). One can observe significant similarities between Figs. 2 and 3 which is natural since both effects have the same physical reason: resonant excitation of surface modes.

To conclude, we report huge near-field enhancements with the use of uniaxial CML and substantial far-field radiation enhancement with randomly placed particles in the vicinity of the CML-vacuum interface.

\section{REFERENCES}

[1] S. I. Maslovski, C. R. Simovski, and S. A. Tretyakov, "Overcoming black body radiation limit in free space: metamaterial superemitter", New Journal of Physics, vol. 18, no. 013034, 2016.

[2] C. A. Valagiannopoulos, et al., "Electromagnetic energy sink", Physical Review B, vol. 92, no. 245402, 2015.

[3] S. D. Gedney, "An anisotropic perfectly matched layer - absorbing medium for the truncation of FDTD lattices", IEEE Transactions Antennas and Propagation, vol. 44, no. 12, pp. 1630-1639, 1996.

[4] E. E. Narimanov and A. V. Kildishev, "Optical black hole: Broadband omnidirectional light absorber", Applied Physics Letters, vol. 95, no. 041106, 2009.

[5] C. A. Valagiannopoulos, et al., "Hyperbolic-metamaterial antennas for broadband enhancement of dipole emission to free space", Journal of Applied Physics, vol. 116, no. 163106, 2014.

[6] C. A. Valagiannopoulos, "On examining the influence of a thin dielectric strip posed across the diameter of a penetrable radiating cylinder", Progress In Electromagnetics Research C, vol. 3, pp. 203-214, 2008. 